\title{
The emerging Haemophilus influenzae serotype a infection and a potential vaccine: Implementation science in action
}

\author{
L Barreto', AD Cox¹, M Ulanova², MG Bruce ${ }^{3}$, RSW Tsang ${ }^{4 *}$
}

\section{Abstract}

Haemophilus influenzae serotype b (Hib) was a major cause of meningitis in children until Hib conjugate vaccine was introduced into the routine infant immunization program and Hib disease in children was almost eliminated. In Alaska, northern Canada and other countries with Indigenous peoples, $\mathrm{H}$. influenzae serotype a (Hia) has emerged as a significant cause of pneumonia, meningitis and septic arthritis especially in children under 24 months of age. A joint government initiative between the Public Health Agency of Canada (PHAC) and the National Research Council of Canada (NRC) was carried out to assess whether an Hia vaccine could be developed for the common good.

The initiative included strategic partnerships with clinician researchers in Thunder Bay, Ontario who provide health services to Indigenous people and the Artic Investigations Program (AIP) of the United States Centers for Disease Control and Prevention (CDC) in Alaska. This government initiated and funded research identified that the development of an Hia vaccine is possible and ongoing surveillance that includes strain characterization is essential to understand the potential spread of Hia in North America and around the world.

\author{
Affiliations \\ ${ }^{1}$ Human Health Therapeutics \\ Portfolio, National Research \\ Council of Canada, Ottawa, ON \\ ${ }^{2}$ Northern Ontario School of \\ Medicine, Lakehead University, \\ Thunder Bay, ON \\ ${ }^{3}$ Arctic Investigations Program, \\ Division of Preparedness \\ and Emerging Infections, US \\ Centers for Disease Control and \\ Prevention (CDC), Anchorage, AK \\ ${ }^{4}$ National Microbiology \\ Laboratory, Public Health Agency \\ of Canada, Winnipeg, MB
}

*Correspondence: raymond. tsang@phac-aspc.gc.ca

Suggested citation: Barreto L, Cox AD, Ulanova M, Bruce MG, Tsang RSW. The emerging Haemophilus influenzae serotype a infection and a potential vaccine: Implementation science in action. Can Commun Dis Rep. 2017;43(5):85-88. https://doi.org/10.14745/ccdr.v43i05a01

\section{Introduction}

Implementation science speaks to the very reason why we do health research: to understand how things work, to test hypotheses, to develop solutions, and to assess effectiveness so we can improve individual and population health. But this process is rarely as straightforward as it seems. The conference proceedings on the Haemophilus influenzae serotype a (Hia) workshop in this issue of the Canada Communicable Disease Report (CCDR) (1) identified the many different types of evidence needed to develop a new vaccine and the challenges still ahead to convert initial research into an approved product. In this editorial, we will explain what Hia is and how it initially emerged, highlight some of the unique aspects involved in developing an Hia vaccine and underscore the importance of ongoing surveillance to observe trends in Hia infections both in North America and around the world.

\section{Background}

Haemophilus influenzae $(H$. influenzae) is a human pathogen that normally resides in the upper respiratory tract, but may occasionally be found in the urogenital mucosa leading to urinary tract, neonatal and obstetric infections (2-4). H. influenzae strains include those that have on their surfaces polysaccharide capsules which divide the strains antigenically into six different capsular serotypes (a to f) and those without capsules (termed non-typeable) (5). Encapsulated strains tend to cause invasive diseases such as meningitis, septicemia, bacteremic pneumonia and septic arthritis, while non-capsulated strains generally cause non-invasive infections such as otitis media, sinusitis and bronchitis.

Hib was a major cause of meningitis in children under the age of five $(6,7)$ until Hib conjugate vaccine was introduced into the routine infant immunization program in the early 1990s. Since that point, Hib disease in children has almost been eliminated (8).

Unfortunately, the declining rates of Hib disease following infant immunization did not completely eliminate invasive $H$. influenzae disease. In the post-Hib vaccine era, non-b serotypes and non-typeable $H$. influenzae strains became more common (9-11). In Alaska, United States (US) (12), and in northern Canada (13-15), as well as in regions with a large proportion of Indigenous people $(16,17)$, Hia has emerged as a significant cause of invasive disease, especially in children under 24 months of age $(18,19)$. Hia causes pneumonia, meningitis and septic arthritis, and it is responsible for a considerable amount of morbidity, life-time disability and mortality $(12,13,18)$. 


\section{Unique aspects of Hia vaccine development}

In this issue of CCDR, Cox and colleagues have identified the critical evidence needed when considering the development of a new vaccine. This evidence includes: surveillance data, laboratory research, pre-clinical studies, regulatory considerations, good manufacturing practice in producing clinical vaccine lots, clinical trial capacity, best practices in public-private partnerships, and more (20). The conference proceedings highlight two unique aspects of Hia vaccine development. From the start, it has been an inter-departmental collaboration within the Government of Canada that included strategic partnerships and second, it has been government-initiated research, with internal funding for both proof-of-concept vaccine research and ongoing surveillance.

\section{Inter-departmental collaboration}

In the early 2000s, the Public Health Agency of Canada (PHAC) and the National Research Council (NRC) of Canada came together to collaborate on the general theme of "expanding vaccine development in Canada". This partnership was formed on the basis of the unique capabilities and expertise of the two different government departments in order to implement scientific solutions for the common good. At the time, laboratory surveillance activities at the National Microbiology Laboratory (NML) had identified a significant percentage of serotype $a$ isolates that were responsible for invasive $H$. influenzae disease (20). Consultation with the Council of Provincial and Territorial Medical Officers of Health and the subsequent review of invasive Hia data from the northern Canadian territories collected via the International Circumpolar Surveillance System confirmed that Hia was a significant emerging pathogen $(21,22)$ causing severe invasive disease for which no vaccine was currently available. Building on the success of the Hib conjugate vaccine developed in the 1980s for control of invasive Hib disease, the similarities of the biology of $\mathrm{Hia}$ and Hib, and the diseases they cause, research was initiated to lay the foundation for developing a Hia conjugate vaccine.

\section{Strategic external partnerships}

The Thunder Bay campus of the Northern Ontario School of Medicine at Lakehead University is situated in Northwestern Ontario and its educational and research activities are affiliated with the Thunder Bay Regional Health Sciences Centre - a 375-bed academic teaching hospital. Both the Northern Ontario School of Medicine and the teaching hospital provide a variety of health services to a population with a significant percentage of Indigenous people. The Arctic Investigations Program (AIP) of the US Centers for Disease Control and Prevention (CDC) located in Anchorage, Alaska has extensive experience in the study of invasive Hib disease beginning in the 1970s before the Hib conjugate vaccine was introduced. Investigators at the AIP had also conducted clinical trials with the Hib conjugate vaccines leading eventually to the implementation of the current Hib-OMV (PRP-OMP) vaccine specifically for American Indigenous children (23).

Each partner in this multi-disciplinary collaboration brings in unique but complementary expertise to the group. The NML of PHAC and the US CDC's AIP provide laboratory surveillance of infectious diseases in the respective countries and NML has an extensive culture collection in a bio-bank for research and development. NRC has a strong tradition in conducting microbial carbohydrate research, extensive experience in protein carbohydrate conjugation technology and has engaged with industry to develop vaccine products. The Northern Ontario School of Medicine has access to a large regional clinical facility and has unique expertise in immunology of infectious diseases and immunoassay for vaccine related issues. The formation of this partnership has been designed with the goal to enhance the success in the development of a Hia conjugate vaccine.

\section{Government funded surveillance and vaccine development work}

Another unique aspect of developing the Hia vaccine is that it has been a government-based initiative. The NML of PHAC identified the potential need for an Hia vaccine through its laboratory surveillance program funded by the Government of Canada. The lead scientist at the NML on this file has knowledge on bacterial vaccines and professional relationships with scientists at the NRC, which opened up collaborative opportunities. A small investment from the Government of Canada allowed scientists at the NRC to purify the Hia capsular polysaccharide and develop the required conjugation reaction to produce a research batch of the Hia conjugate vaccine. Immunogenicity studies in laboratory animals have confirmed that the Hia conjugate vaccine can induce bactericidal antibodies, which is a recognized surrogate marker for protective immunity against $H$. influenzae. Hence, studies to date have provided the pre-clinical proof of concept that the Hia conjugate vaccine approach is likely to be as effective as the Hib conjugate vaccine.

Current epidemiological surveillance has revealed that the population most at risk of developing severe invasive Hia diseases are Indigenous children under the age of five and immunocompromised Indigenous adults living in North America and other regions. Much effort has gone into consulting the most affected communities. In Canada, two workshops (including the workshop with proceedings being published in this issue of (CDR) have been conducted that include participants from Indigenous communities and investigators at the Northern Ontario School of Medicine, who continue to engage the Indigenous communities in their research.

\section{Conclusion}

Using evidence to inform practice is a complex undertaking. In the case of vaccine development, evidence to demonstrate burden of illness, laboratory studies, consultations with public health stakeholders, those affected by the disease, regulatory experts and industry partners are the critical components in the process. Yet in the case of Hia vaccine, two unique and additional elements are also notable: the fact that government funding and research identified the problem; and that a government partnership led to a proof-of-concept for a vaccine which facilitated the engagement of others in further developing this solution. The evolving nature of infectious microbes is likely to continue to change the epidemiology of invasive $H$. influenzae 
disease. Therefore, continued surveillance that includes strain characterization is essential to follow the potential spread of $\mathrm{Hia}$ in North America and around the world and, perhaps in the future, to document its decline in response to a new vaccine.

\section{Authors' statement}

All authors (LB, ADC, MU, MGB and RSWT) had input into the concept of this editorial. LB and RSWT prepared the first draft and all authors contributed to the final version with comments and suggestions.

\section{Disclaimer}

The findings and conclusions in this article are those of the authors and do not necessarily represent the official positions of the Public Health Agency of Canada or the United States Centers for Disease Control and Prevention.

\section{Conflict of interest}

ADC, MGB, and RSWT have no conflicts of interest to declare.

MU holds an Investigator Initiated Research Grant from Pfizer for an unrelated study. LB is a Senior Scientific Advisor with Inventprise/InventVac, Redmond, Washington/Vancouver, British Columbia and a Strategic Advisor to NEOMED-LABS, Montreal, Quebec; and he has worked with the NRC Vaccine and Immune Therapeutics Group (HHT) from 2011 to 2015 and as a consultant for NRC in relation to Hia vaccine development. He worked with Sanofi Pasteur from 1988 to 2010 on Hib and combination vaccine development.

\section{Acknowledgements}

We thank Dr. Rainer Engelhardt, Dr. John Spika of PHAC and Dr. Jim Richards of NRC for implementation of the initial phase of this project, Dr. Thomas Hennessy and Dr. Karen Rudolph of US CDC-Alaska for their continued support, Dr. William McCready and Dr. Eli Nix at Northern Ontario School of Medicine, Dr. Lakshmi Krishnan, Dr. Wei Zou, Frank St. Michael, Chantelle Cairns, Dean Williams and Rhonda Kuo Lee of NRC for their support of the Hia project and Dr. Patricia Huston for suggesting the structure for this manuscript.

\section{References}

1. Cox A, Barreto L, Ulanova M, Bruce MG, Tsang RSW on behalf of the Conference contributors. Developing a vaccine for Haemophilus influenzae serotype a: Proceedings of a workshop. Can Commun Dis Rep. 2017;43(5):89-95. Available from: http://www.phac-aspc.gc.ca/publicat/ccdrrmtc/17vol43/dr-rm43-05/ar-2-eng.php.

2. Slack MPE. Haemophilus. In: Borriello SP, Murray PR, Funke $G$ eds. Topley and Wilson's microbiology and microbial infections, 10th ed. Vol 2. Bacteriology. London, UK: Edward Arnold Ltd.; 2006. p.1692-1718.

3. Gabre-Kidan T, Lipsky BA, Plorde JJ. Haemophilus influenzae as a cause of urinary tract infections in men. Arch Inter Med. 1984;144:1623-1627.

4. Wallace Jr. RJ, Baker CJ, Quintones FJ, Hollis DG, Weaver RE, Wiss K. Nontypable Haemophilus influenzae (biotype 4) as a neonatal, maternal, and genital pathogen. Rev Infect Dis. 1983;5:123-36.

5. Pittman M. Variation and type specificity in the bacterial species Haemophilus influezae. J Exp Med. 1931;53:471-92.

6. Shapir ED, Ward JJ. The epidemiology and prevention of disease caused by Haemophilus influenzae type b. Epidemiol Rev 1991;13:113-142.

7. Ulanova M, Tsang RSW. Invasive Haemophilus influenzae disease: Changing epidemiology and host-parasite interactions in the 21st century. Infect Gen Evol. 2009;9:594605.

8. Bath S, Bisgard K, Murphy T, Shutt K, Rosenstein N, Ohuabunwo C, and the Centers for Disease Control and Prevention (CDC). Progress towards elimination of Haemophilus influenzae type $b$ invasive disease among infants and children---United States, 1998-2000. MMWR Morb Mortal Wkly Rep. 2002;51:234-7.

9. Urwin G, Krohn JA, Deaver-Robinson K, Wenger JD, Farley MM, and the Haemophilus influenzae Study Group. Invasive disease due to Haemophilus influenzae serotype f: clinical and epidemiologic characteristics in the $\mathrm{H}$. influenzae serotype b vaccine era. Clin Infect Dis. 1996;22:1069-76.

10. Sadeghi-Aval P, Tsang RSW, Jamieson F, Ulanova M. Emergence of non-type $b$ encapsulated Haemophilus influenzae as a cause of pediatric meningitis in Northwestern Ontario. Can J Infect Dis Med Microbiol. 2013;24:13-6.

11. Van Eldere J, Slack MPE, Ladhani S, Cripps AW. Nontypeable Haemophilus influenzae, an under-recognised pathogen. Lancet Infect Dis. 2014;14:1281-92.

12. Bruce MG, Zulz T, DeByle C, Singleton R, Hurlburt D, Bruden $D$, et al. Haemophilus influenzae serotype a invasive disease, Alaska, USA, 1983-2011. Emerg Infect Dis. 2013;19:932-7.

13. Boisvert AA, Moore D. Invasive disease due to Haemophilus influenzae type a in children in Canada's north: A priority for prevention. Can J Infect Dis Med Microbiol. 2015;26:291-2.

14. Tsang RSW, Li YA, Mullen A, Baikie M, Whyte K, Shuel M, et al. Laboratory characterization of invasive Haemophilus influenzae isolates from Nunavut, Canada, 2000-2012. Int J Circumpolar Health. 2016;75:297-8.

15. Bruce MG, Deeks SL, Zulz T, Navarro C, Palacios C, Cases CC, et al. Epidemiology of Haemophilus influenzae serotype a, North American Arctic, 2000-2005. Emerg Infect Dis. 2008;14:48-55.

16. Millar EV, O'Brien KL, Watt JP et al. Epidemiology of invasive Haemophilus influenzae type a disease among Navajo and White Mountain Apache children, 1988-2003. Clin Infect Dis. 2005;40:823-30.

17. Kelly L, Tsang RSW, Morgan A, Jamieson FB, Ulanova M. Invasive disease caused by Haemophilus influenzae type 
a in Northern Ontario First Nations communities. J Med Microbiol. 2011;60:384-90.

18. Ulanova M, Tsang RSW. Haemophilus influenzae serotype a as a cause of serious invasive infections. Lancet Infect Dis. 2014; 14: 70-82.

19. Tsang RSW, Bruce MG, Lem M, Barreto L, Ulanova M. A review of invasive Haemophilus influenzae disease in the Indigenous populations in North America. Epidemiol Infect. 2014;142:1344-54.

20. Tsang RSW, Mubareka S, Sill ML, Wylie J, Skinner S, Law DKS. Invasive Haemophilus influenzae in Manitoba, Canada, in the postvaccination era. J Clin Microbiol. 2006;44:1530-5.

21. Bruce MG, Deeks SL, Zulz T, Navarro C, Palacios C, Cases $C C$, et al. Epidemiology of Haemophilus influenzae serotype a, North American Arctic, 2000-2005. Emerg Infect Dis. $2008 ; 14: 48-55$.

22. Desai S, Lee YA, Zulz T, Bruce M. Monitoring invasive bacterial diseases in the North American Arctic via the International Circumpolar Surveillance Project. Can Commun Dis Rep. 2015;41(3):52-4. Available from: http://www.phacaspc.gc.ca/publicat/ccdr-rmtc/15vol41/dr-rm41-03/surv-2eng.php.

23. American Academy of Pediatrics. Summary of infectious diseases, Section 3, Haemophilus influenzae infections, pp. 345-352. In: Pickering LK, Baker CJ, Kimberlin DW, Long SS, eds. Red Book: 2010 Report of the Committee on Infectious Diseases. Elk Groove Village, IL: American Academic of Pediatrics; 2012.

Did you know? CCDR publishes peer-reviewed articles every issue, in addition to the theme.

\section{SUBMIT YOUR PAPER TO THE CCDR EDITORIAL OFFICE TODAY!}

- It's FREE to submit and publish (no submission, publishing, or article processing charges)

- Theme correlation not required

- Go online and visit the CCDR Submit a Manuscript page for more information.
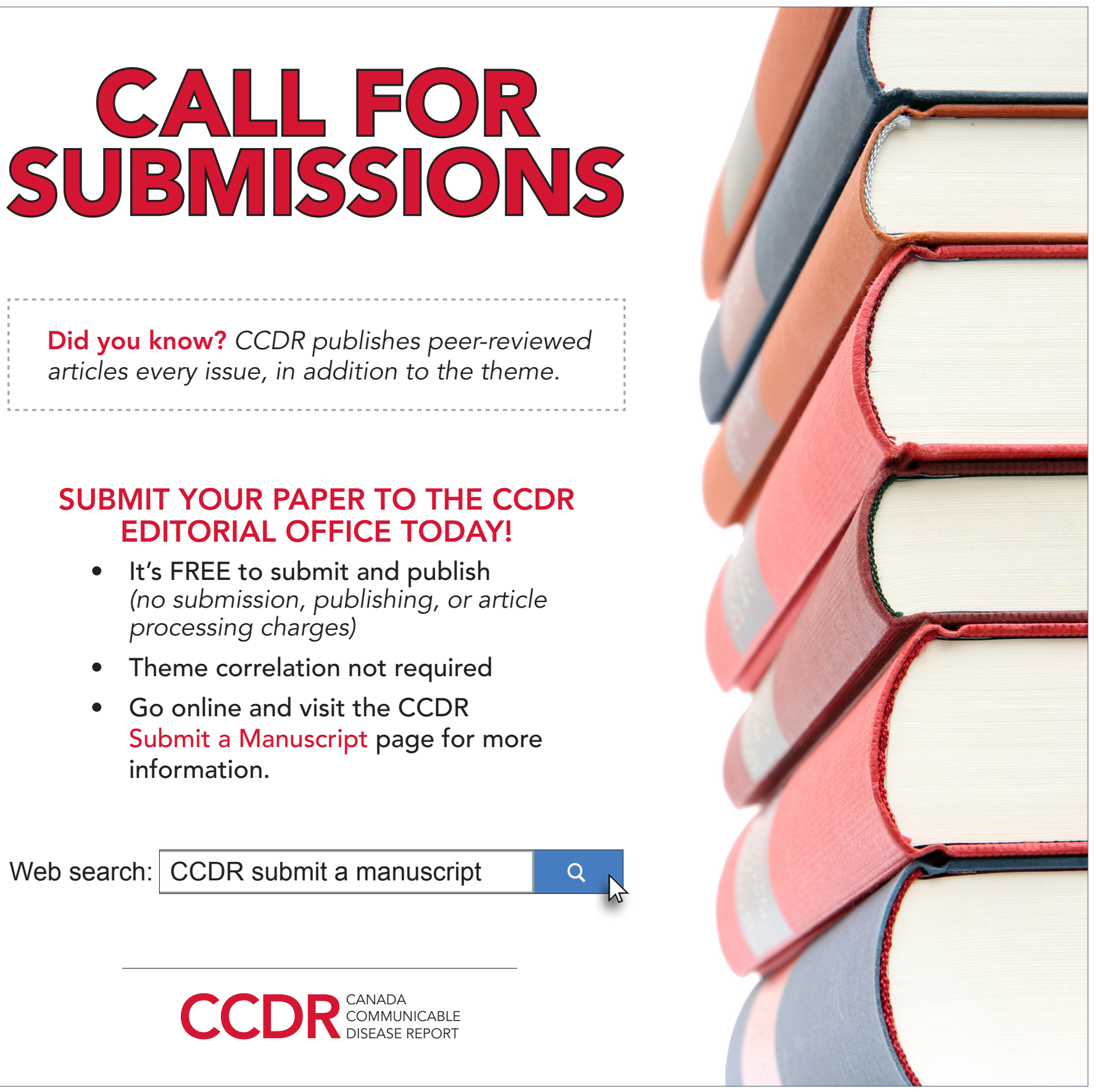